\title{
Prevalencia de la enfermedad de Chagas en puérperas y transmisión congénita en una zona endémica del Perú
}

\author{
Carlos Alberto Mendoza Ticona, ${ }^{1}$ Eleazar Córdova Benzaquen, ${ }^{2}$ \\ Jenny Ancca Juárez, ${ }^{3}$ Joselito Saldaña Díaz, ${ }^{3}$ Aymé Torres Choque, ${ }^{3}$ \\ Renato Velásquez Talavera, ${ }^{2}$ Juan de los Ríos Álvarez, ${ }^{4}$ Jesús Saldaña Díaz, ${ }^{4}$ \\ Silvia Vega Chirinos ${ }^{5}$ y Ronald Sánchez Pérez ${ }^{4}$
}

Forma de citar Mendoza Ticona CA, Córdova Benzaquen E, Ancca Juárez J, Saldaña Díaz J, Torres Choque A, Velásquez Talavera $R$, et al. Prevalencia de la enfermedad de Chagas en puérperas y transmisión congénita en una zona endémica del Perú. Rev Panam Salud Publica. 2005;17(3):147-53.

RESUMEN Objetivos. Determinar la prevalencia de anticuerpos contra Trypanosoma cruzi en puérperas y la posible transmisión congénita de la enfermedad de Chagas en Arequipa, Perú, una zona donde esta enfermedad es endémica.

Métodos. Se estudió a las puérperas que dieron a luz entre diciembre de 2001 y julio de 2002 en tres hospitales (dos urbanos y uno rural) y cuatro centros de salud (tres rurales y uno urbano) del departamento de Arequipa, Perú. El estudio serológico comprendió el tamizaje de todas las puérperas para detectar anticuerpos contra T. cruzi mediante inmunofluorescencia indirecta (IFI); la prueba de inmunoadsorción enzimática (ELISA) y la titulación de anticuerpos IgG por IFI se usaron como pruebas confirmatorias. A las puérperas con seropositividad y a sus recién nacidos se les realizó la prueba de detección de anticuerpos IgM mediante IFI y se evaluó la presencia de infección mediante xenodiagnóstico (evaluada a los 30 y 60 días) y el micrométodo de Freilij. Los resultados se analizaron según la presencia del vector y de casos de enfermedad de Chagas en los lugares de nacimiento y de residencia de las puérperas. Dos neonatólogos evaluaron clínicamente a los recién nacidos para detectar anomalías y signos de enfermedad de Chagas congénita.

Resultados. La prevalencia general de enfermedad de Chagas en las 3000 puérperas estudiadas fue de 0,73\%; fue mayor en dos centros de salud ubicados en zonas rurales $(2,2 \%$ en El Pedregal y 4,1\% en La Joya) $(\mathrm{P}=0,018)$ y la enfermedad estuvo asociada con el contacto directo previo con el vector $(\mathrm{P}<0,05)$ y con el haber nacido en una zona considerada endémica $(\mathrm{P}<0,01)$. Cuatro de las 20 puérperas con seropositividad $(20 \%)$ tuvieron resultados positivos en el xenodiagnóstico. Ninguna conocía su estado de portadora de la infección y no se observaron síntomas o signos característicos de la enfermedad de Chagas aguda o crónica. En ninguna puérpera se detectaron anticuerpos IgM y solo un neonato (nacido de una madre sin parasitemia) presentó un título de IgM de 1/8, pero en los controles posteriores no se detectaron anticuerpos IgM o IgG. No se detectaron parásitos en la sangre de los neonatos por ninguno de los dos métodos empleados. De los 20 neonatos evaluados, uno tenía microcefalia

\footnotetext{
1 Universidad Peruana Cayetano Heredia, Lima, Perú. La correspondencia debe ser dirigida a Carlos Alberto Mendoza Ticona, Instituto de Medicina Tropical Alexander von Humboldt, Av. Honorio Delgado 460, Ingeniería, San Martín de Porras,
}

Lima, Perú. Correo electrónico: mendozalberto@ hotmail.com; 24273@upch.edu.pe

2 Universidad Nacional de San Agustín, Arequipa, Perú.
3 Laboratorio de Referencia Regional, Dirección de Salud de Arequipa, Arequipa, Perú.

4 Hospital Regional Honorio Delgado, Arequipa, Perú. Instituto Nacional de Salud, Lima, Perú. 
y hepatoesplenomegalia y aunque tenía anticuerpos específicos IgG contra T. cruzi al nacer, estos desaparecieron a los dos meses; el crecimiento y el desarrollo de los demás recién nacidos fueron normales.

Conclusión. La prevalencia de enfermedad de Chagas en puérperas del departamento de Arequipa, Perú, es baja. No se encontraron casos de transmisión congénita intrauterina. Se recomienda diseñar estudios de detección prenatal que permitan evaluar a un mayor número de madres y en el que participen también las mujeres que dan a luz en sus domicilios.

Palabras clave Enfermedad de Chagas, transmisión vertical de enfermedad, prevalencia, Perú.

La enfermedad de Chagas o tripanosomiasis sudamericana constituye un problema de salud pública en la mayoría de los países latinoamericanos. Según la Organización Mundial de la Salud, de 16 a 18 millones de personas están infectadas con este parásito entre la frontera norte de México y la parte más austral de la Argentina y Chile (1).

Se han descrito tres formas de transmisión vectorial: la intradomiciliaria, la peridomiciliaria y la silvestre. La primera es la forma más frecuente de transmisión en América Latina y se produce cuando hay contacto de la piel o las mucosas de personas con las heces o la orina de insectos hematófagos infectados con Trypanosoma cruzi. El principal vector domiciliario es Triatoma infestans, conocido en el suroccidente peruano como "chirimacha", y es el único insecto vector demostrado en esta región hasta el momento. Los reservorios más importantes que intervienen en la transmisión vectorial son el hombre, los gatos, los perros, los roedores y, en el sur peruano, el cuy o cobayo (Cavia porcella).

Otra vía frecuente de transmisión es la parenteral, por la transfusión de sangre de personas portadoras. Aunque solo una parte de los receptores de sangre infectada adquieren la enfermedad, esta proporción puede variar mucho y se han informado cifras de $18 \%$ a $25 \%$ en Brasil, Argentina y Chile, y hasta de $48 \%$ en Bolivia (2).

Una tercera forma de transmisión de la enfermedad de Chagas es la materno-fetal, estudiada recientemente por Carlier y colaboradores (3). Contrariamente a observaciones previas en casos de enfermedad de Chagas congénita, estos autores no encontraron infiltración de los tripomastigotos en los vellos placentarios, sino en los fibroblastos coriónicos y en el mesénquima subamniótico, lo que podría indicar que la transmisión materno-fetal ocurre por la vía coriónica, sin invasión directa del trofoblasto. Esta transmisión puede ocurrir en cualquier momento de la gestación, pero es más frecuente durante el tercer trimestre. Debido a la migración de mujeres en edad fértil desde las zonas rurales a las ciudades, la transmisión congénita puede convertirse en un problema de salud también en las zonas urbanas $(2,4)$.

En el Perú existen dos regiones endémicas: la suroccidental (departamentos de Arequipa, Tacna, Moquegua e Ica) y la selva norte (departamentos de Cajamarca, Amazonas y San Martín) (figura 1). En estos departamentos viven alrededor de 1970000 habitantes, los cuales pueden considerarse en riesgo de adquirir esta infección (5). En 1997 se inició la vigilancia de la enfermedad de Chagas en Perú y se estableció su notificación obligatoria en todo el país. La tasa nacional acumulada de personas infectadas por T. cruzi en el Perú en los últimos años es de 7,29 por 100000 habitantes y se calcula que en las áreas endémicas viven 24170 personas infectadas por este parásito (5). Se estima que $5 \%$ de los enfermos padecen formas agudas $\mathrm{u}$ oligosintomáticas, mientras que el restante $95 \%$ tiene formas crónicas de la enfermedad; $13 \%$ de los enfermos son niños menores de 5 años de edad (5). Los esfuerzos por erradicar el vector no han logrado detener la propagación de la enfermedad y de hecho el vector se ha extendido de los valles interandinos del suroccidente peruano a las principales ciudades de esa región, entre ellas Arequipa, Moquegua y Tacna (5).
En América Latina, la prevalencia de esta enfermedad en gestantes varía de $2 \%$ a $51 \%$ en centros urbanos y de $23 \%$ a $81 \%$ en zonas rurales de regiones endémicas (1). La tasa de transmisión congénita varía según la región geográfica: en Argentina es de 0,7 a $8,8 \%(6,7)$, en Paraguay de 10,5\% (8), en Chile de $2,1 \%$ a $9,8 \%(9,10)$, en Brasil de $1,6 \%$ (11) y en Bolivia de $2 \%$ a $21 \%$ (12). En el Perú no se conoce la prevalencia de la infección por T. cruzi en mujeres gestantes de regiones endémicas y aún no se han documentado casos congénitos.

El objetivo del presente trabajo fue determinar la prevalencia de anticuerpos contra T. cruzi en puérperas y la posible transmisión congénita de la enfermedad de Chagas en Arequipa, Perú, una zona donde esta enfermedad es endémica.

FIGURA 1. Regiones del Perú con enfermedad de Chagas endémica, 2004

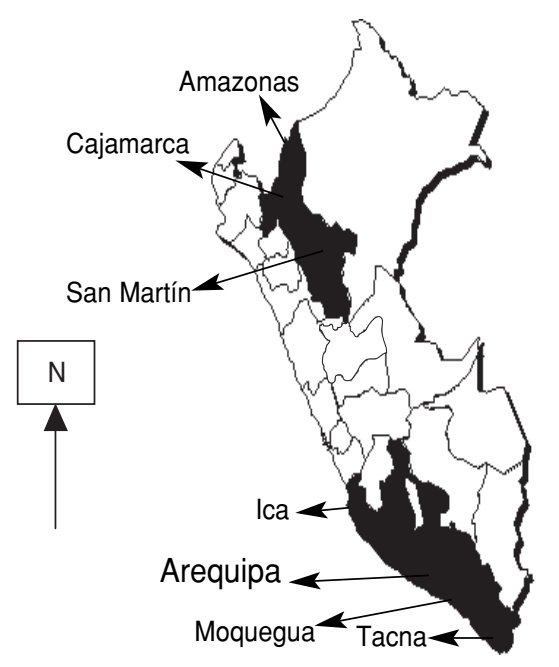




\section{MATERIALES Y MÉTODOS}

El estudio se llevó a cabo entre diciembre de 2001 y julio de 2002 en tres instalaciones hospitalarias del Ministerio de Salud: el Hospital Regional Honorio Delgado, el Hospital Apoyo Goyeneche, ambos de la ciudad de Arequipa (urbanos), el Hospital Apoyo Aplao, de la provincia de Castilla en el departamento de Arequipa (rural), así como en cuatro centros de salud con capacidad para atender partos ubicados en zonas endémicas para la enfermedad de Chagas: Corire, El Pedregal, La Joya (ubicados en zonas rurales del departamento de Arequipa) y Jacobo D. Hunter (en la ciudad de Arequipa).

Se evaluó a todas las puérperas que dieron a luz en los centros seleccionados. Se coordinó con el Programa Nacional de Control de Enfermedades de Transmisión Sexual y Sida para, una vez obtenido el consentimiento oral de rigor, utilizar la muestra de sangre periférica que se extrae a todas las puérperas inmediatamente después del parto para el tamizaje de la sífilis, que se realiza de forma gratuita y obligatoria, según disposiciones del Ministerio de Salud del Perú.

A las puérperas se les preguntó su lugar de nacimiento y de residencia, datos que se agruparon según su pertenencia a zonas con enfermedad de Chagas endémica (distritos donde se hubieran informado casos de infección humana por T. cruzi, según datos recientes de la Región de Salud de Arequipa) o a zonas no endémicas. También se les preguntó si tuvieron contacto directo con el vector, es decir, si detectaron la presencia de triatómidos en la casa que habitaban o hubieran habitado, para lo cual se les mostraron ejemplares de $T$. infestans en sus cinco estadios. A las puérperas con anticuerpos contra T. cruzi se les preguntó acerca de las posibles vías de infección, si sabían que eran portadoras de la infección y si habían notado alguno de los síntomas asociados con la enfermedad de Chagas. Dos neonatólogos evaluaron clínicamente a los recién nacidos para detectar anomalías y signos de enfermedad de Chagas congénita.
La infección en las puérperas se determinó mediante la prueba de inmunofluorescencia indirecta (IFI) para IgG (Calbiochem, EUA). Las muestras con título de 1/20 o mayores se confirmaron mediante inmunoadsorción enzimática (ELISA) para IgG (Organon Teknika, Argentina) validado con sueros conocidos procedentes del Laboratorio de Parasitología de la Facultad de Medicina de la Universidad Nacional de San Agustín de Arequipa, Perú. Las muestras se consideraron reactivas si el título de anticuerpos de la clase IgG según la prueba de IFI era de 1/40 o mayor, y si el resultado del ELISA era positivo según los criterios del fabricante.

A las puérperas cuyas muestras resultaron reactivas se les pidió su consentimiento por escrito para realizar pruebas que permitieran confirmar la transmisión congénita de la infección a sus hijos. Para ello se determinó la presencia de anticuerpos específicos mediante las pruebas de IFI para IgG (Calbiochem, EUA) e IgM (Sigma Chemical, EUA). La detección de parasitemia en los neonatos y puérperas se realizó mediante las técnicas de xenodiagnóstico. También se aplicó el micrométodo de Freilij para los recién nacidos. El xenodiagnóstico consistió en colocar sobre la piel del brazo del recién nacido una caja con 10 ninfas de $T$. infestans en estadío III nacidas y criadas en el laboratorio, sin infección por T. cruzi (en el caso de las puérperas se emplearon dos de esas cajas). A los $30 \mathrm{y}$ 60 días se analizó el contenido intestinal de los insectos en busca de tripomastigotos de T. cruzi. Adicionalmente se aplicó el micrométodo descrito por Freilij y col. (13) ${ }^{6}$ para buscar directamente la presencia de parásitos en la sangre capilar tomada del talón de cada recién nacido con ocho capilares. Las muestras de los neonatos se tomaron en el hospital o en el domicilio durante los primeros 15 días de vida. El criterio diagnóstico de transmisión congénita de la enfermedad de Chagas fue la detección del parásito en la sangre del recién nacido, ya fuera mediante xenodiagnóstico o por el micrométodo.

\footnotetext{
6 En la referencia 13 este apellido figura como "Feilij", aunque debería ser "Freilij".
}

Las pruebas serológicas y parasitológicas se realizaron en los Laboratorios de Parasitología de la Universidad Nacional de San Agustín de Arequipa y de la Dirección de Salud de Arequipa. El control de la calidad de los resultados serológicos se llevó a cabo en el Laboratorio de Parasitología del Instituto Nacional de Salud, en Lima. Para las pruebas serológicas y parasitológicas se siguieron las Guías de Laboratorio para el Diagnóstico de la Enfermedad de Chagas, del Instituto Nacional de Salud del Perú (14).

El estudio contó con la autorización del Comité de Ética del Instituto Nacional de Salud del Perú, como requisito indispensable para la aprobación presupuestaria por el Proyecto Vigía.

Los datos se presentan en valores totales y porcentuales, con medidas de tendencia central y de dispersión para una mejor comprensión de los resultados. Para evaluar las diferencias encontradas en la distribución de las prevalencias se aplicó la prueba de ji al cuadrado o la prueba exacta de Fisher. El nivel de significación utilizado fue de 0,05 .

\section{RESULTADOS}

En total se estudió a 3000 puérperas de 13 a 46 años (edad media: 25,6 años). De ellas, 2419 (80,63\%) procedían de zonas con enfermedad de Chagas endémica y $1475(49,17 \%)$ manifestaron haber tenido contacto directo con el vector (cuadro 1).

Durante el tamizaje inicial, 51 (1,7\%) de las 3000 muestras resultaron reactivas con títulos de anticuerpos IgG por IFI de al menos $1 / 20$. Sin embargo, solo 22 de ellas fueron reactivas por ELISA para IgG y tuvieron títulos por IFI de 1/40 o mayores, por lo que la prevalencia fue de $0,73 \%$.

Las 22 puérperas positivas presentaron títulos de anticuerpos IgG contra T. cruzi por IFI entre $1 / 40$ y 1/1 280, y ninguna tenía anticuerpos de la clase IgM según la prueba de IFI. De las 20 puérperas a las que se les realizó la prueba de xenodiagnóstico, en 4 (20\%) se detectó el parásito en la sangre. No obstante, aunque sus hijos recién naci- 
CUADRO 1. Características generales de las puérperas estudiadas. ${ }^{a}$ Arequipa, Perú, diciembre de 2001 a julio de 2002

\begin{tabular}{|c|c|c|}
\hline Variable & No. & $\%$ \\
\hline \multicolumn{3}{|l|}{ Lugar de residencia ${ }^{b}$} \\
\hline Zonas endémicas en el departamento de Arequipa ${ }^{c}$ & 2413 & 80,43 \\
\hline Zonas no endémicas en el departamento de Arequipa & 577 & 19,23 \\
\hline Zonas endémicas fuera del departamento de Arequipa & 6 & 0,20 \\
\hline Zonas no endémicas fuera del departamento de Arequipa & 4 & 0,14 \\
\hline \multicolumn{3}{|l|}{ Lugar de nacimientob } \\
\hline Zonas endémicas en el departamento de Arequipac & 1235 & 41,17 \\
\hline Zonas no endémicas en el departamento de Arequipa & 599 & 19,97 \\
\hline Zonas endémicas fuera del departamento de Arequipa & 75 & 2,5 \\
\hline Zonas no endémicas fuera del departamento de Arequipa & 1091 & 36,37 \\
\hline \multicolumn{3}{|l|}{ Establecimiento de salud } \\
\hline Hospital Regional Honorio Delgado & 1808 & 60,27 \\
\hline Hospital Goyeneche & 920 & 30,67 \\
\hline Hospital Aplao & 79 & 2,63 \\
\hline Centro de Salud La Joya & 49 & 1,63 \\
\hline Centro de Salud Pedregal & 90 & 3,0 \\
\hline Centro de Salud Corire & 3 & 0,1 \\
\hline Centro de Salud J. D. Hunter & 51 & 1,7 \\
\hline \multicolumn{3}{|l|}{ Tipo de parto } \\
\hline Vaginal & 2642 & 88,07 \\
\hline Cesárea & 358 & 11,93 \\
\hline Contacto directo con Triatoma infestans & 1475 & 49,17 \\
\hline
\end{tabular}

${ }^{a} N=3$ 000; edad promedio: 25,6 años (desviación estándar: $\pm 6,17$; intervalo: de 13 a 46 años).

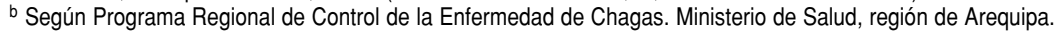

c Se consideraron zonas endémicas los distritos en los que se habían notificado casos de personas infectadas por Trypanosoma cruzi.

dos tuvieron títulos altos de anticuerpos según la prueba de IFI para IgG $(1 / 640)$ y el ELISA, en ninguno se encontró el parásito.

Según los datos epidemiológicos de las 22 puérperas con seropositividad (cuadro 2), la prevalencia de anticuerpos específicos contra $T$. cruzi fue mayor en dos centros de salud ubicados en la zona rural ( $2,2 \%$ en El Pedregal y $4,1 \%$ en La Joya) $(P=0,018)$. La prevalencia fue mayor en las puérperas que nacieron en zonas endémicas $(P<$ $0,01)$ y en las que refirieron haber tenido contacto directo con el vector $(P=$ $0,025)$. Ninguna mostró síntomas o signos característicos de la enfermedad de Chagas aguda o crónica. De las 22 puérperas con seropositividad, 16 afirmaron haber tenido contacto directo con el vector ( 9 en zonas rurales y 7 en la ciudad de Arequipa) y 6 de ellas confirmaron la presencia intradomiciliaria del vector en el momento del estudio. Todas negaron haber recibido transfusiones de sangre y desconocían su estado de portadoras de la infección.
La mayoría de los neonatos nacieron de parto eutócico, a término, con medidas antropométricas adecuadas para su edad gestacional. Solo uno de ellos fue prematuro y tenía microcefalia y hepatoesplenomegalia; este paciente tuvo un resultado positivo a la prueba de citomegalovirus (detección de IgM) y sus anticuerpos de la clase IgG contra T. cruzi desaparecieron a los dos meses de vida.

Se logró captar a 20 de los 22 niños nacidos de madres serorreactivas (la información de residencia de los dos restantes no era correcta). Las muestras de 19 de los recién nacidos estudiados (95\%) fueron reactivas según la prueba de IFI para IgG, con títulos entre $1 / 20$ y 1/640 en el momento de la captación para el estudio, mientras que 18 (90\%) tenían anticuerpos específicos de la clase IgG, según los resultados de la prueba de ELISA. Un neonato tuvo una reacción positiva a IgM con un título de $1 / 8$, pero esos anticuerpos desaparecieron al mes siguiente y no se encontraron parásitos en la madre. En ninguno de los 20 recién nacidos evaluados se encontraron parásitos ni por el micrométodo de Freilij ni por el xenodiagnóstico a los 30 y a los 60 días.

\section{DISCUSIÓN}

La prevalencia de anticuerpos contra el agente causal de la enfermedad de Chagas en puérperas del departamento de Arequipa $(0,73 \%)$ fue similar a la observada en el estado brasileño de Londrina (0,9\%) en el año 2000 (15). Sin embargo, estas prevalencias son inferiores a las encontradas en mujeres gestantes de otras regiones endémicas de América Latina, como la zona noroccidental de Argentina (5,5\%) y Santiago de Chile $(3,7 \%)(6,10)$, y muy inferiores a las observadas en Paraguay $(15,4 \%)$ y Bolivia $(51,0 \%)(8,12)$.

La prevalencia encontrada no se distribuyó homogéneamente, ya que en dos de los centros rurales estudiados alcanzó valores entre 2,2\% y $4,1 \%$, comparables con los observados en Chile y Argentina, y fue mayor entre las puérperas nacidas en zonas endémicas y entre las que tuvieron contacto directo con el vector. No se encontró ninguna asociación entre una mayor prevalencia y el hecho de radicar en zonas endémicas, lo que indica que la infección materna podría haberse adquirido a edades tempranas y que son los fenómenos migratorios los que provocan la aparición de casos de Chagas en zonas sin vectores ni enfermos, creándose así el riesgo de transmisión de la enfermedad, como lo comprobaron Mallimaci y col. en Ushuaia, la ciudad más austral del mundo (4).

Recientemente se informó de que el índice de infestación domiciliaria con $T$. infestans en zonas urbanas de la ciudad de Arequipa podía llegar a 47,8\%, lo que demuestra la abundancia del vector en la región estudiada (16). El hecho de que la mitad de las puérperas que participaron en este estudio hayan identificado a la "chirimacha" y afirmaran haber tenido contacto directo con ese vector reafirma esta apreciación. Sin embargo, el reducido número de personas con anticuerpos contra $T$. cruzi contrasta con esos hallazgos. En 
CUADRO 2. Características epidemiológicas de las puérperas con anticuerpos contra T. cruzi. ${ }^{a}$ Arequipa, Perú, diciembre de 2001 a julio de 2002

\begin{tabular}{|c|c|c|c|}
\hline Variable & No. & $\%$ & $P$ \\
\hline \multicolumn{4}{|l|}{ Según el lugar de residencia } \\
\hline Zona endémica ${ }^{b}$ & $17 / 2419$ & 0,70 & \multirow[t]{2}{*}{$0,59^{e}$} \\
\hline Zona no endémica & $5 / 581$ & 0,86 & \\
\hline \multicolumn{4}{|l|}{ Lugar de nacimiento } \\
\hline Zona endémicab & 19/1 310 & 1,45 & \multirow[t]{2}{*}{$<0,001^{\dagger}$} \\
\hline Zona no endémica & $3 / 1690$ & 0,17 & \\
\hline \multicolumn{4}{|c|}{ Casos según el establecimiento de salud en que se atendió } \\
\hline Hospital Regional Honorio Delgadoc & $12 / 1808$ & 0,66 & \multirow[t]{8}{*}{$0,018^{f}$} \\
\hline Hospital Goyeneche ${ }^{c}$ & $6 / 920$ & 0,65 & \\
\hline Centro de Salud La Joya ${ }^{d}$ & $2 / 49$ & 4,08 & \\
\hline Centro de Salud Pedregald & $2 / 90$ & 2,22 & \\
\hline Hospital Aplao ${ }^{d}$ & $0 / 79$ & 0 & \\
\hline Centro de Salud Corire ${ }^{d}$ & $0 / 3$ & 0 & \\
\hline Centro de Salud J.D. Hunter ${ }^{c}$ & $0 / 51$ & 0 & \\
\hline Total & $22 / 3000$ & 0,73 & \\
\hline \multicolumn{4}{|l|}{ Contacto directo con Triatoma infestans } \\
\hline Sí & $16 / 1475$ & 1,08 & \multirow[t]{2}{*}{$0,025^{f}$} \\
\hline No & 6/1 525 & 0,39 & \\
\hline \multicolumn{4}{|l|}{ Condiciones epidemiológicas } \\
\hline Presencia del vector en la vivienda actual & $6 / 22$ & 27.00 & \\
\hline Transfusiones de sangre & $0 / 22$ & 0 & \\
\hline Conocimiento previo de la seropositividad & $0 / 22$ & 0 & \\
\hline
\end{tabular}

${ }^{a} N$ = 22; edad promedio: 25,5 años (desviación estándar: $\pm 7,34$; intervalo: de 15 a 42 años).

b Se consideraron zonas endémicas los distritos en los que se habían notificado casos de personas infectadas por Trypanosoma cruzi.

c Establecimiento de salud en la ciudad de Arequipa.

d Establecimiento de salud en la zona rural, fuera de la ciudad de Arequipa.

e Prueba exacta de Fisher.

${ }^{\dagger}$ Prueba de ji al cuadrado.

un estudio retrospectivo realizado entre 1991 y 2001 en el servicio de pediatría de los hospitales Honorio Delgado y Goyeneche, solo se detectaron 13 casos de enfermedad de Chagas en fase aguda, de los cuales 2 fallecieron (17). Aunque puede haber un subregistro de casos, estos resultados indican que el número de personas con la enfermedad clínica característica es más bajo de lo esperado, incluso en zonas donde es frecuente encontrar el vector y el parásito. Para encontrar una explicación a este fenómeno contradictorio se requieren estudios adicionales que profundicen en las particularidades epidemiológicas, clínicas, fisiopatológicas y genéticas tanto del hospedero como del vector y del parásito.

El porcentaje de casos reactivos con título de IgG de $1 / 20$ o mayor según la IFI fue de $1,7 \%$, similar a la prevalencia de 1,59\% observada en donantes de sangre de los hospitales de la región sur del país (18). Debido a que los casos reactivos encontrados durante el tamizaje de donantes de sangre con sistemas de ELISA para IgG de diversos fabricantes no se confirmaron con otras pruebas diagnósticas, no se descarta que la prevalencia real en ese grupo de población sea mucho menor.

De las 22 puérperas que tenían anticuerpos contra T. cruzi, 18 nacieron en el departamento de Arequipa; de ellas, 12 nacieron en la ciudad de Arequipa y 14 residían allí en el momento del diagnóstico. Esto apoya la hipótesis de que la infección por este parásito se está extendiendo a zonas urbanas, tanto por la migración de personas portadoras hacia las ciudades como por la aparición de triatómidos en zonas urbanas y periurbanas de ciudades importantes del sur peruano, como Arequipa, Moquegua y Tacna.

Debido al reducido número de puérperas reactivas encontradas, no fue posible evaluar el riesgo de transmisión congénita en un mayor número de re- cién nacidos. Sin embargo, los resultados del estudio serológico de los recién nacidos, la ausencia de parásitos en ellos y el buen desarrollo intrauterino de 19 de los 20 neonatos estudiados corroboran los hallazgos de otras investigaciones realizadas en regiones urbanas de Argentina $(6,19,20)$, Chile $(10,21)$ y Brasil $(22)$, en las cuales se encontró que la proporción de casos asintomáticos era elevada (de 60\% a $90 \%$ ), pero que la tasa de transmisión congénita era baja (3). Sin embargo, la morbilidad en niños nacidos de madres con seropositividad en Bolivia fue de hasta $50 \%$, con una tasa de mortalidad de $2 \%$ a $14 \%$ de los neonatos infectados (3).

En cuanto al Perú, solo se conoce un estudio de transmisión congénita en el que se evaluó mediante xenodiagnóstico a 320 recién nacidos en los hospitales Honorio Delgado, Aplao y el centro de salud La Joya en 1985. Al igual que en la presente investigación, este autor tampoco encontró casos de transmisión congénita a pesar de que seis de los niños estudiados nacieron de madres con resultados serológicos positivos y en cuatro de ellos también se detectaron anticuerpos contra T. cruzi (23).

Tomando en cuenta la elevada sensibilidad y especificidad tanto del xenodiagnóstico (14) como del micrométodo de Freilij (13) para detectar la parasitemia durante la fase aguda, los resultados obtenidos permiten confirmar que no hubo transmisión congénita (intrauterina) de T. cruzi en los 20 neonatos evaluados. Sin embargo, no es posible descartar la posible transmisión de la infección en el momento del parto, ya que los estudios de detección temprana de parasitemia - como el realizado aquí- no tienen la sensibilidad suficiente para detectar esos casos. Por lo tanto, es necesario evaluar otras técnicas de diagnóstico, como la reacción en cadena de la polimerasa o la detección de anticuerpos específicos mediante antígenos recombinantes para la fase aguda y la evaluación serológica de los niños después de los 9 meses de vida, siempre que se garantice que no haya triatómidos en sus viviendas $(3,8)$. En el presente estudio no se realizó ese seguimiento, ya que no se podía garantizar que en las vi- 
viendas de los niños no hubiera vectores. Por otra parte, los resultados obtenidos confirman que la detección de anticuerpos de la clase IgM contra $T$. cruzi no es suficiente para determinar la transmisión congénita de la enfermedad de Chagas.

Teniendo en cuenta la baja prevalencia de la enfermedad de Chagas en gestantes y la ausencia de transmisión congénita en la zona estudiada, se recomienda que el Programa Nacional de Control de la Enfermedad de Chagas del Perú concentre su atención y recursos en el control de la transmisión horizontal, es decir, en combatir el vector y reforzar el tamizaje de los donantes en los bancos de sangre. El control de la transmisión vertical pasaría a ser una prioridad solo después de eliminar la transmisión vectorial, proceso que aún avanza lentamente en el Perú (18).
En este trabajo no se evaluó a las madres que dieron a luz en sus domicilios, que son las que por lo general tienen mayores carencias económicas y mayor riesgo de padecer de la enfermedad de Chagas. Se recomienda diseñar estudios más amplios de prevalencia de esta enfermedad en mujeres gestantes, que permitan determinar desde el punto de vista de la salud pública y de la relación costo-beneficio la pertinencia de establecer un sistema de control prenatal para esta enfermedad en las dos regiones endémicas del país.

Agradecimientos. Este estudio contó con el apoyo técnico y financiero del Proyecto VIGIA “Enfrentando las Amenazas de las Enfermedades Infecciosas Emergentes y Reemergentes", del Mi- nisterio de Salud del Perú y de la Agencia de los Estados Unidos para el Desarrollo Internacional (USAID). Los autores agradecen al señor Justo Ayta y al doctor Juan Cornejo por su invaluable colaboración. Agradecen además a los doctores Carlos Seas y Eduardo Gotuzzo, del Instituto de Medicina Tropical Alexander von Humboldt de la Universidad Peruana Cayetano Heredia, Sergio Sosa-Estani, del Ministerio de Salud de Argentina, e Yves Carlier, de la Universidad Libre de Bruselas, Bélgica, por la revisión y sugerencias hechas al manuscrito. Los autores también quieren dejar testimonio de su agradecimiento al personal de la Dirección de Salud de Arequipa, de los hospitales Honorio Delgado, Goyeneche y Aplao, y de los centros de salud El Pedregal, La Joya, Jacobo D. Hunter y Corire por su apoyo constante.

\section{REFERENCIAS}

1. World Health Organization. Control of Chagas disease. Report of a WHO Expert Committee. Geneva: WHO; 1991. (Technical Report Series WHO/RSD/91.811).

2. Brener Z, Andrade Z, Barral-Neto M. Trypanosoma cruzi e doença de Chagas. 2. ${ }^{a}$ ed. Rio do Janeiro: Guanabara-Koogan; 2000.

3. Carlier Y, Torrico F. Congenital infection with Trypanosoma cruzi: from mechanisms of transmission to strategies for diagnosis and control. Rev Soc Bras Med Trop. 2003;36:767-71.

4. Mallimaci MC, Sijvarger C, Dates A, Álvarez M, Sosa-Estani S. Seroprevalencia de la enfermedad de Chagas en Ushuaia, Argentina, una zona sin triatominos. Rev Panam Salud $\mathrm{Pu}-$ blica. 2001;9(3):169-71.

5. Perú, Ministerios de Salud. Reunión Macroregional Sur para el Plan de Control de la Enfermedad de Chagas y Eliminación del Triatoma infestans. Lima: MINSA; 1999.

6. Blanco S, Segura E, Cura E, Chuit R, Tulian L, Flores-Igarbarino $\mathrm{G}$, et al. Congenital transmission of Trypanosoma cruzi: an operational outline for detecting and treating infected infants in North-Western Argentina. Trop Med Inter Health. 2000;5:293-301.

7. Contreras S, Fernández M, Agüero F, Desse J, Orduña T, Martino O. Enfermedad de ChagasMazza congénita en Salta, Argentina. Rev Soc Bras Med Trop. 1999;32:633-6.

8. Russomando G, de Tomassone M, de Guillen I, Acosta N, Vera N, Almiron M, et al. Treatment of congenital Chagas disease diagnosed and followed up by the polimerasa chain reaction. Am J Trop Med Hyg. 1998;59:487-91.

9. Lorca M, Thiermann E. Diagnóstico serológico de las infecciones congénitas por Try- panosoma cruzi. Rev Chil Pediatr. 1991;62: 337-44.

10. Muñoz $P$, Thiermann E, Atias $M$, Acevedo C. Enfermedad de Chagas sintomática en recién nacidos y lactantes. Rev Chil Pediatr.1992;63: 192-202.

11. Bittencourt AL, Mota E, Ribeiro R, Fernandes LG, de Almeida PR, Sherlock I, et al. Incidence of congenital Chagas' disease in Bahia, Brazil. J Trop Pediatr. 1985;31:242-8.

12. Azogue E, la Fuente C, Darras C. Congenital Chagas' disease in Bolivia: epidemological aspects and pathological findings. Trans R Soc Trop Med Hyg. 1985;79:176-80.

13. Feilij H, Muller L, Gonzáles-Cappa S. Direct micromethod for diagnosis of acute and congenital Chagas' disease. J Clin Microbiol. 1983;18:327-30.

14. Perú, Ministerio de Salud. Normas de laboratorio para el diagnóstico de la tripanosomiasis americana. Lima: Instituto Nacional de Salud; 2000.

15. Reiche EM, Morimoto HK, Farias GN, Hisatsugu KR, Geller L, Gomes AC, et al. Prevalência de tripanossomíase americana, sífilis, toxoplasmose, rubéola, hepatite $B$, hepatite $\mathrm{C}$ e da infecção pelo virus da inmunodeficiência humana, avaliada por intermédio de testes serológicos, em gestantes atendidas no período de 1996 a 1998 no Hospital Universitário Regional Norte do Londrina Paraná. Rev Soc Bras Med Trop. 2000;33:519-27.

16. Mendoza A, Córdova E, Cornejo JY, Linares O. Aspectos epidemiológicos de la enfermedad de Chagas en una localidad urbana de la ciudad de Arequipa. Rev Peruana Enf Infec Trop. 2003;2:49.
17. Mendoza A, Córdova E, Guerra FY, de los Ríos J. Enfermedad de Chagas: aspectos epidemiológicos, clínicos y terapéuticos en pacientes pediátricos de los hospitales Honorio Delgado y Goyeneche de Arequipa, años 1991-2001. Rev Peruana Enf Infec Trop. 2003; 2:19.

18. República del Perú, Ministerio de Salud. Informe técnico sobre el control de la enfermedad de Chagas en el Perú, 2000. X Reunión de la Comisión Intergubernamental del Cono Sur para la Eliminación de Triatoma infestans y la Interrupción Transfusional de la Tripanosomiasis Americana. Lima: MINSA-DGSP; 2001.

19. Zaidenberg M, Segovia A. Enfermedad de Chagas congénita en la ciudad de Salta, Argentina. Rev Inst Med Trop São Paulo. 1993; 35:35-43.

20. Freilij H, Altcheh J. Congenital Chagas' disease: diagnostic and clinical aspects. Clin Infect Dis. 1995;21:551-5.

21. Howard J. La enfermedad de Chagas congénita. Santiago: Universidad de Chile; 1962.

22. Bittencourt A. Possible risk factors for vertical transmission of Chagas' disease. Rev Inst Med Trop São Paulo. 1992;34:403-8.

23. Manrique-Zegarra M. Transmisión congénita de la enfermedad de Chagas en el Hospital Regional Honorio Delgado, CS La Joya y Hospital Aplao [tesis de bachiller médico cirujano]. Arequipa, Perú: Universidad Nacional de San Agustín; 1985.

Manuscrito recibido el 28 de junio de 2004. Aceptado para publicación, tras revisión, el 16 de noviembre de 2004. 


\section{ABSTRACT \\ The prevalence of Chagas' disease in puerperal women and congenital transmission in an endemic area of Peru}

Objectives. To determine the prevalence of antibodies against Trypanosoma cruzi in puerperal women and to assess possible congenital transmission of Chagas' disease in the department of Arequipa, Peru, where the disease is endemic.

Methods. Women who had given birth between December 2001 and July 2002 in three hospitals (two urban and one rural) and four health centers (three rural and one urban) of the department of Arequipa, Peru, were studied. The serological study included screening all the puerperal women in order to detect antibodies against T. cruzi through indirect immunofluorescence (IIF), with confirmatory testing done with enzyme-linked immunosorbent assay (ELISA) testing and the titration of immunoglobulin $\mathrm{G}$ (IgG) antibodies by IIF. IIF tests to screen for immunoglobulin M (IgM) antibodies were done with the seropositive women and their newborns, and infection was evaluated through xenodiagnosis (evaluated at 30 and 60 days) and the direct micromethod of Freilij et al. The results were analyzed in terms of the presence of the vector and of cases of Chagas' disease in the places where the puerperal women had been born and where they were living. Two neonatologists clinically evaluated the newborns in order to detect abnormalities and signs of congenital Chagas' disease.

Results. The overall prevalence of Chagas' disease in the 3000 puerperal women studied was $0.73 \%$. Prevalence was highest in two health centers located in rural areas ( $2.2 \%$ in El Pedregal and $4.1 \%$ in La Joya) $(P=0.018)$. The disease was associated with previous direct contact with the vector $(P<0.05)$ and with having been born in an area considered endemic $(P<0.01)$. Four $(20 \%)$ of the 20 seropositive puerperal women were also positive by xenodiagnosis. However, none of the women was aware of her infectious carrier state, and none showed the characteristic symptoms or signs of acute or chronic Chagas' disease. IgM antibodies were not detected in any of the puerperal women. One neonate (whose mother did not have evidence of parasitemia) presented an IgM titer of $1 / 8$, but in later controls neither IgM nor IgG antibodies were detected. Parasites were not detected in the blood of the neonates by either of the two testing methods used. Of the 20 neonates evaluated, one presented microcephaly and hepatosplenomegaly; although the child had specific IgG antibodies against T. cruzi at birth, the antibodies were not present at the age of two months. The growth and development of the other 19 newborns were normal.

Conclusions. The prevalence of Chagas' disease in puerperal women of the department of Arequipa, Peru, is low. No cases of intrauterine congenital transmission were found. We recommend carrying out studies on prenatal detection that evaluate more mothers and in which women who give birth at home also participate.

Keywords: Chagas disease; disease transmission, vertical; prevalence; Peru.

\title{
Free Online Training in Public Health
}

The Center for Public Health Preparedness of the state of North Carolina (NCCPHP), in the United States of America, is offering more than 100 free on-line training modules on a wide range of areas of public health. All of the modules are in English. Many of the modules uses audio tape or videotape lectures that last from 30 to 60 minutes, and then allow persons who want to test their new knowledge or to receive free continuing education credits to take a short on-line examination.

The general categories of the training modules range from epidemiology, outbreak investigations, and emerging and reemerging diseases to surveillance, biostatistics, and bioterrorism. Specific epidemiology modules include how to read the epidemiologic literature, common measures and statistics in the epidemiologic literature, data analysis and causal inference, and population screening. Modules in biostatistics include commonly used statistical tests, confidence intervals, an introduction to linear regression, and inference for two-way tables.

The modules could be useful to public health professionals who want to expand their knowledge and skills, to public health students, and to other audiences. The NCCPHP also offers free materials that trainers can use, such as PowerPoint slide presentations for a class, workshop, or other training session.

\author{
Information: \\ Lorraine Alexander \\ North Carolina Center for Public Health Preparedness \\ E-mail: nccphpw3@sph.unc.edu \\ Web site: http://www.sph.unc.edu/nccphp/training/training_list/
}

